\title{
Are DSM and logic not on good terms?
}

Peter Dome, Zoltan Demeter, Xenia Gonda and Zoltan Rihmer

\section{Summary}

We would like to draw attention to the fact that the recently published DSM-5 (and also its predecessor, DSM-IV) contains annoying errors that are mainly logical in nature. These mistakes are undoubtedly a result of inadvertence, rather than either conceptual (professional) disagreements between authors/editors or shortage of scientific data for appropriate circumscription of diagnostic categories. The good news is that since these errors are mainly logical ones, they can be recognised and repaired.

\section{Declaration of interest}

None.

\section{Copyright and usage}

(c) The Royal College of Psychiatrists 2015.
Peter Dome (pictured) is a research psychiatrist in the Department of Clinical and Theoretical Mental Health at Semmelweis University and at the National Institute of Psychiatry and Addictions in Budapest. Zoltan Demeter is a research assistant in the Department of Clinical and Theoretical Mental Health at Semmelweis University. Xenia Gonda is an associate professor in the Department of Clinical and Theoretical Mental Health at Semmelweis University, a researcher at the National Institute of Psychiatry and Addictions, Budapest and at the MTA-SE Neuropsychopharmacology and Neurochemistry Research Group. Zoltan Rihmer is Professor of Psychiatry in the Department of Clinical and Theoretical Mental Health at Semmelweis University and in the National Institute of Psychiatry and Addictions, Budapest.

DSM is one of the most widely used nosological systems for establishing psychiatric diagnoses with a more than 60-year history behind it. ${ }^{1}$ Despite heroic efforts preceding publication of successive editions of DSM, prima facie recognisable logical errors may be found even in the recently published fifth edition. ${ }^{1}$ Some of these were undoubtedly caused by the inattentiveness of the authors/editors (rather than by professional/conceptual disagreements between them).

Our aim was, by thorough reading of the chapters 'Schizophrenia spectrum and other psychotic disorders', 'Bipolar and related disorders' and 'Depressive disorders' in DSM-5 and 'Schizophrenia and other psychotic disorders' and 'Mood disorders' in DSM-IV, ${ }^{2}$ to identify clinically relevant changes in the new edition of DSM. Two authors (P.D. and Z.D.) conducted the primary analysis of the text and discussed the issues raised with the senior authors (X.G. and Z.R.). We have only presented in this paper those errors that were deemed to exist and considered important by all authors.

\section{Findings}

\section{1 - Criterion for brief psychotic disorder}

See criterion A for brief psychotic disorder (p. 94): 'Presence of one (or more) of the following symptoms. At least one of these must be (1), (2), or (3).' There then follow four symptoms. According to the rules of logic, the fourth symptom has no relevance to the establishment of a brief psychotic disorder diagnosis. This criterion was not misleading in DSM-IV $(\text { p. 304) })^{2}$ (it was the same but without the phrase 'At least one of these must be (1), (2), or (3)'). What kind of relevant data may have led to the change of the operable criterion of brief psychotic disorder in DSM-IV? Even if such data (i.e. data suggesting the superfluity of the fourth symptom for brief psychotic disorder) exists, why did the authors decide to add a phrase to criterion A that renders the whole criterion illogical instead of deleting the fourth symptom?

\section{2 - Criterion for schizophrenia and schizophreniform disorder}

A scientific text should be as concise as possible, but that is not always true of DSM. For example, see criterion D for schizophrenia (p. 99) or the identical criterion C for schizophreniform disorder (p. 97). These criteria are intended to exclude schizoaffective disorder and depressive/bipolar disorders with psychotic features in this way: 'either 1) no major depressive or manic episodes have occurred concurrently with the active-phase symptoms, or 2) if mood episodes have occurred during active-phase symptoms, they have been present for a minority of the total duration of the active and residual periods of the illness. ${ }^{1}$ Similar phrasing was already present in DSM-IV (pp. 285-286). ${ }^{2}$ Since 'mood episodes' as a group consists of major depressive and manic episodes, the authors should have considered applying Ockham's razor and cancelled point (1) accordingly. However, even if it is very unlikely, it is conceivable that the intention of the authors in point (2) was to permit the occurrence of hypomanic episode (as the third, and final, type of mood episode in DSM-5) during the active-phase symptoms of schizophrenia. Yet, the second sentence of criterion E for hypomanic episode (p. 125) excludes the possibility that hypomania may coexist with psychotic symptoms and ultimately with schizophrenia or schizophreniform disorder. ${ }^{1}$

\section{3 - Criterion for manic episode}

See criterion A for manic episode in the DSM-5 (p. 124). ${ }^{1}$ This criterion makes it clear that increased goal-directed activity or energy' should be present during the episode. If so, why does a conceptually very similar (almost identical) symptom appear in criterion B (sixth item) as a possible but not necessary symptom of a manic episode? This contradiction was not present in $\operatorname{DSM-IV~(p.~332).~}{ }^{2}$

\section{4 - Criterion for delusional disorder}

See criterion B for delusional disorder (p. 90): 'Criterion A for schizophrenia has never been met'. Criterion A for schizophrenia is as follows (p. 99): 'Two (or more) of the following, each present for a significant portion of time during a 1-month period (or less if successfully treated). At least one of these must be (1), (2), or 
(3): 1. Delusions; 2. Hallucinations . ... ' Now let us look at criterion A for delusional disorder (p. 90) 'The presence of one (or more) delusions with a duration of 1 month or longer' and the note for criterion B for delusional disorder (p. 90) 'Hallucinations, if present, are not prominent and are related to the delusional theme." The duration of hallucination(s) is not limited in delusional disorder so they may be present for longer than a 1-month period and delusion(s) should be present by definition for longer than a month in delusional disorder. The theme of hallucinations is not limited in schizophrenia (i.e. hallucination(s) in schizophrenia may be related to the theme of delusion(s)). It may therefore be the case that delusion(s) and not prominent hallucination(s) related to the delusional theme coexist for more than a month in delusional disorder, thereby fulfilling criterion A for schizophrenia. However, although the concept of delusional disorder (and the phrasing of criterion $\mathrm{A}$ and the note for criterion B; p. 301) and schizophrenia (p. 285) are somewhat different in DSM-IV, the problem is the same as it is in the fifth edition.

\section{5 - Diagnostic features of brief psychotic disorder}

See the last paragraph of 'diagnostic features' for brief psychotic disorder (p. 95): 'In addition to the five symptom domain areas identified in the diagnostic criteria . . . ? ${ }^{1}$ It is obvious that the phrase 'five symptom domain areas' was transferred from identical 'diagnostic features' for schizophreniform disorder (p. 98) and schizophrenia (p. 101). ${ }^{1}$ However, there is a little hitch: in contrast to schizophrenia and schizophreniform disorder, brief psychotic disorder has only four symptoms (and not five).

\section{6 - Criterion for substance/medication-induced bipolar and related disorder}

See criterion A for DSM-5 substance/medication-induced bipolar and related disorder (p. 142). ${ }^{1}$ It is obvious that this criterion is a derivation of A criterion for hypomanic/manic episodes (p. 124) but their exact time criteria (4 and 7 days respectively) were replaced with the dubious term 'persistent'. (However, we may suppose that 'persistent' does not indicate a period longer than the 7 days criterion for a manic episode). The second part of criterion A for substance/medication-induced bipolar and related disorder (begins after 'irritable mood') perhaps reflects the authors' intention of permitting episodes 'with mixed features' (p. 149) in this disorder. In addition, B criteria for hypomanic/ manic episodes were omitted when criteria for substance/ medication-induced bipolar and related disorder were established. Furthermore, an important coding note of the corresponding DSM-IV diagnosis, substance-induced mood disorder, that permits one to diagnose substance-induced mood disorder only in those cases where 'mood symptoms are in excess of those usually associated with the intoxication or withdrawal syndrome ... (p. 374) $)^{2}$ disappeared from the fifth edition. As a final result of these changes, we may/should diagnose substance/medicationinduced bipolar and related disorder, for example, in the case of a patient (with a negative history for non-substance-induced bipolar and related disorder (criterion C) and without delirium (criterion D)) with a period of a few days of markedly elevated mood and impairments in school functioning (criterion $\mathrm{E}$ ) coexisting with, and as a result of, first (regular) cannabis use resulting in intoxication (criterion B).

\section{7 - Definition of postpartum onset of brief psychotic disorder}

Finally, see the definition of 'postpartum onset' of brief psychotic disorder (p. 94). ${ }^{1}$ This specifier states that the postpartum period consists inter alia of the time of pregnancy. It is a radically new interpretation of the term postpartum.

Examples five to seven differ somewhat from the first four examples because these last three are not primarily logical errors. However, all seven examples are similar in that inadvertence can be supposed to have contributed to their occurrence.

\section{Discussion}

Given that psychiatry and DSM are frequent targets of attacks in scientific and non-scientific forums, a more fastidious approach to preparing/editing/proofreading DSM is recommended in order to avoid at least those issues that do not result from the inherent features of psychiatry (i.e. which are not entirely conceptual ones) ${ }^{3-7}$ According to our knowledge, DSM was mainly criticised previously because of conceptual issues. However, numerous non-conceptual errors may further damage its reputation.

\footnotetext{
Peter Dome, MD, PhD, Department of Clinical and Theoretical Mental Health Semmelweis University, Budapest, and National Institute of Psychiatry and Addictions, Budapest; Zoltan Demeter, Department of Clinical and Theoretical Mental Health, Semmelweis University, Budapest; Xenia Gonda, MA, PharmD, PhD, Department of Clinical and Theoretical Mental Health, Semmelweis University, Budapest, National Institute of Psychiatry and Addictions, Budapest, and MTA-SE Neuropsychopharmacology and Neurochemistry Research Group, Budapest: Zoltan Rihmer, MD, PhD, DSc, Department of Clinical and Theoretical Mental Health Zoltan Rihmer, MD, PhD, DSC, Department of Clinical and Theoretical Mental Health,
Semmelweis University, Budapest, and National Institute of Psychiatry and Addictions, Budapest, Hungary

Correspondence: Peter Dome, Semmelweis University, Kútvölgyi út 4, Budapest H-1125, Hungary. Email: dome_peter@yahoo.co.uk

First received 24 Jun 2014, final revision 9 Nov 2014, accepted 17 Feb 2015
}

\section{Funding}

P.D. and X.G. are recipients of the János Bolyai Research Scholarship of the Hungarian Academy of Sciences.

\section{References}

1 American Psychiatric Association. Diagnostic and Statistical Manual of Mental Disorders (5th edn) (DSM-5). APA, 2013.

2 American Psychiatric Association. Diagnostic and Statistical Manual of Mental Disorders (4th edn) (DSM-IV). APA, 1994.

3 Lieberman JA. Psychiatry on the scientific spectrum. New York Times 2013; 29 May (http://www.nytimes.com/2013/05/30/opinion/psychiatry-on-thescientific-spectrum.html?_r=0).

4 Reese H. The real problems with psychiatry. Atlantic 2013; 2 May (http://www.theatlantic.com/health/archive/2013/05/the-real-problemswith-psychiatry/275371/).

5 Frances A. ICD, DSM and the Tower of Babel. Aust NZ J Psychiatry 2014; 48: $371-3$.

6 Frances AJ, Nardo JM. ICD-11 should not repeat the mistakes made by DSM-5. Br J Psychiatry 2013; 203: 1-2.

7 Reardon S. NIH rethinks psychiatry trials. Nature 2014; 507: 288. 